\title{
Optimized Indoor Positioning for static mode smart devices using BLE
}

\author{
Quang Huy Nguyen, Princy Johnson, Trung Thanh Nguyen and Martin Randles \\ Faculty of Engineering and Technology, Liverpool John Moores University \\ Liverpool, UK, L3 3AF \\ Q.H.Nguyen@2016.ljmu.ac.uk, \{P.Johnson, T.T.Nguyen, M.J.Randles\}@ljmu.ac.uk
}

\begin{abstract}
Bluetooth Low Energy (BLE) technology and BLE-based devices such as iBeacons have become popular recently. In this work, an optimized indoor positioning approach using BLE for detecting a smart device's location in an indoor environment is proposed. The first stage of the proposed approach is a calibration stage for initialization. The Received Signal Strength Indicator (RSSI) is collected and pre-processed for a stable outcome, in the second stage. Then the distance is estimated by using the processed RSSI and calibrated factors in the third stage. The final stage is the position estimation using the outputs from the previous steps. The positioning technique, which is an improved Least Square estimation is evaluated against the other well-known techniques such as, Trilateration-Centroid, classic Least Square Estimation in estimating the user's location in the 2D plane. Experimental results show that our proposed approach has promising results by achieving an accuracy of positioning within 0.2 to $0.35 \mathrm{~m}$.
\end{abstract}

Keywords - Localization; Indoor Location; Bluetooth Low Energy; iBeacon, RSSI.

\section{INTRODUCTION}

Indoor positioning is the ability to locate people or objects inside a building. The focus and need for this ability has greatly increased recently. Indoor positioning offers four main applications categorized as proximity marketing /advertising, way-finding/ navigation, search/ requesting help, and asset or people tracking. Hence, this topic is a huge attraction for researchers. At present, the well-established positioning systems, such as Global Navigation Satellite System (GNSS) including the famous Global Positioning System (GPS) can only provide good performance in outdoor environment. The signal from satellites is blocked by walls, people and other objects. Moreover, the acceptable error range for an outdoor positioning might be larger than an indoor environment. This means that the available GPS chips on the market cannot adapt to the requirements of indoor positioning. There are several challenges an indoor positioning system need to address: accuracy, complexity, cost, scalability, power consumption, noise and interference [1-2].

There are several well-known technologies currently available for Indoor Positioning such as Wi-Fi, BLE, RFID or Visual Light Communication (VLC). We have chosen BLE [3] as the main technology for our research work due to its several advantages over other techniques. BLE or Bluetooth 4.0 is the new standard of Bluetooth since 2010. It is based on simple communication process and offers low energy consumption [3]. The BLE communication process consists of two main stages: advertising via transmitters, and connection between transmitters and receivers. In practice, this process allows an application in the mobile devices to listen and collect information from the BLE transmitters. We have selected iBeacon technology [4] from Apple as transmitters for this research. The principle involved is that the beacons periodically advertise data packets to be picked by the mobile devices. The Received Signal Strength Indicator (RSSI) then can be measured and used to estimate the distance between the beacons and the mobile devices. Once the distance is known, the position of the devices can be calculated using appropriate techniques such as Least Square Estimation [5-6] or Trilateration-Centroid [7]. These methods require very accurate and stable RSSI and distance measurement to achieve good results. Further research is required in this area of improving the position estimation techniques.

In this paper, a novel optimized approach for indoor positioning from RSSI based estimation using BLE is proposed. In our approach, the environment factor and received power factor are calibrated using linear regression. We have evaluated the effectiveness of the traditional filters such as Kalman filter, Gaussian filter, feedback filter and average filter in combating the instability due to unreliable RSSI feed. Following this, the distance is calculated and location of the user is estimated using an improved version of Least Square Estimation. Finally, we have compared the performance of our technique with that of other methods. For this stage of research, we have considered the user's smart device as static with a Line-Of-Sight communication. Our next stage of research will evaluate the technique in real-life environment that includes noise from various sources, and mobility added to the user's device.

The rest of this paper is organized as follows: section II describes our proposed technique; section III discusses the experiment and results and section IV is the conclusion and future work. 


\section{PROPOSED METHOD}

The different stages of the proposed optimized indoor positioning approach are shown in Fig.1.

\section{A. Propagation model and factor calibration}

The most commonly used indoor propagation model is the log-normal shadowing. It represents and simplifies the relationship between RSSI, distance and "noise" term as shown in equation (1) below:

$$
\text { RSSI }=A-10 \times \eta \times \log _{10}\left(d / d_{0}\right)
$$

In this formula, $d$ is the distance between transmitter and receiver; $A$ is the RSSI at a reference distance $d_{0} ; \eta$ is the environment factor or "noise" factor. Using reference distance as 1 meter, from formula (1) we see that the distance and RSSI is dependent on $\mathrm{A}$ and $\eta$ as given by (2):

$$
\mathrm{RSSI}=\mathrm{A}-10 \times \eta \times \log _{10}(\mathrm{~d})
$$

Under the complexity of different indoor environments such as multipath, human body, interference etc., and devices condition such as battery life, antenna direction, choosing a generic set value of $\mathrm{A}$ and $\eta$ can cause errors in ranging estimation later. Hence, these factors should be calibrated and corrected.

In the given area where the localization of a device is to be calibrated, $[\mathrm{d}]_{\mathrm{n}}$ is a set of $\mathrm{n}$ known reference distances $\left[d_{1}, d_{2} \ldots d_{n-1}, d_{n}\right]$ and $[R S S I]_{n}$ is a set of $n$ pre-measured RSSI $\left[\mathrm{RSSI}_{1}, \mathrm{RSSI}_{2} \ldots \mathrm{RSSI}_{\mathrm{n}-1}, \mathrm{RSSI}_{\mathrm{n}}\right.$ ]. This is shown in Fig. 2. Using linear regression method, the relationship between $\mathrm{A}$ and $\eta$ can be expressed by the formula (3):

$$
[\mathrm{RSSI}]_{\mathrm{n}}=\eta \times[\mathrm{d}]_{\mathrm{n}}+\mathrm{A}
$$

Solving (3) gives the value of $A$ and $\eta$ for a specific area and a device.

\section{B. RSSI processing}

The RSSI is vulnerable to the environment. It strongly depends on multipath propagation. Various other conditions, such as transceiver direction, battery level, obstacles, device model, etc. will also affect the RSSI value. Hence, smoothing and stabilizing the RSSI signal is critical to achieve a satisfactory result in indoor positioning. Fig. 3 shows the RSSI over time at $1 \mathrm{~m}, 5 \mathrm{~m}$ and $10 \mathrm{~m}$.

Several filters including averaging filter, feedback filter [8], Gaussian filter and Kalman filter [10] were considered for smoothing the RSSI in this work.

\section{1) Averaging filter}

This is the simplest method to smooth the RSSI signal. Multiple RSSI values are measured for one node and the average value is calculated using (4) given below:

$$
\overline{\mathrm{RSSI}}=\frac{1}{\mathrm{~m}} \sum_{\mathrm{i}=1}^{\mathrm{m}} \mathrm{RSSI}_{\mathrm{i}}
$$

where $\overline{\mathrm{RSSI}}$ is the mean RSSI; $m$ is the number of measurements and RSSI ${ }_{i}$ is the RSSI of the ith beacon.

\section{1) Feedback filter}

Feedback filter was described in [8] and [9] to eliminate the large differences in the measured value of
RSSI. Its principle is to add a weighted value $\alpha$ to the RSSI to correct RSSI values. In addition, feedback filter considers the previous RSSI measurement to make sure the RSSI can be smoothed. Formula (5) shows this solution:

$\mathrm{RSSI}_{\text {smoothed }}=\alpha \times \mathrm{RSSI}_{\mathrm{k}}+(1-\alpha) \times \mathrm{RSSI}_{\mathrm{k}-1}$

In this equation, $\mathrm{k}$ is the current measurement, whereas $\mathrm{k}-1$ is the previous measurement.

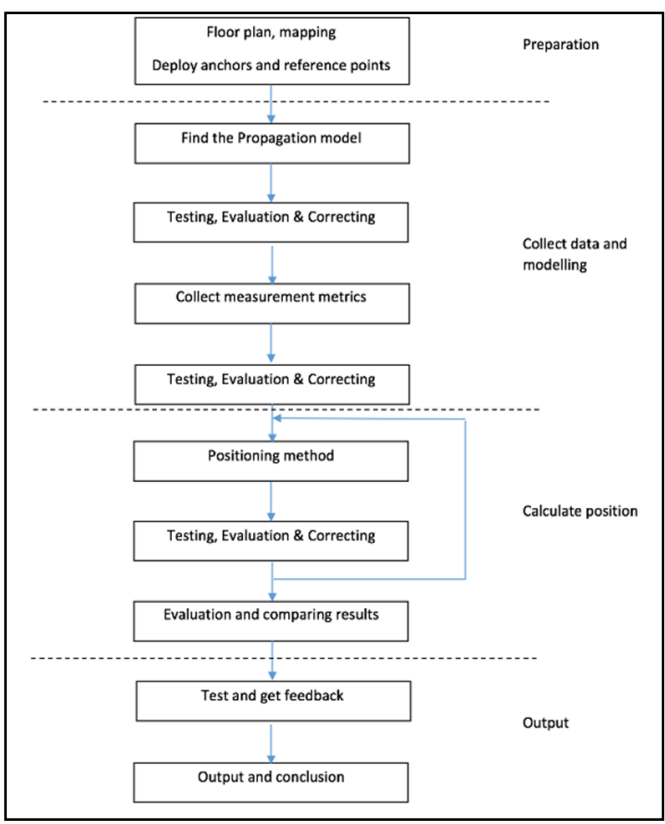

Fig. 1. Optimized Indoor Positioning overview

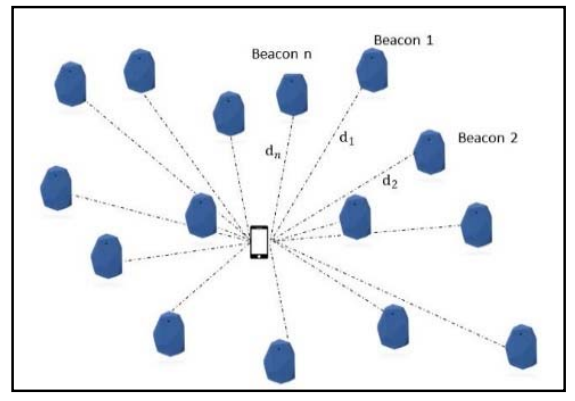

Fig. 2. Calibration phase measurements

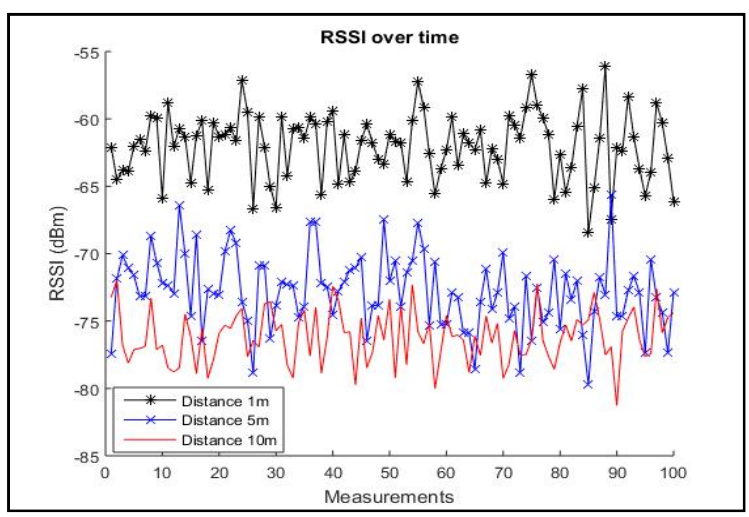

Fig. 3. RSSI over time 


\section{2) Gaussian filter}

Gaussian filter is designed to eliminate noise following the normal distribution. Its formula for RSSI is represented by (6):

$$
f(R S S I)=\frac{1}{\sqrt{2 \pi \sigma^{2}}} e^{\frac{-(\text { RSSI }-\mu)^{2}}{2 \sigma^{2}}}
$$

where $\mu$ is the mean $\overline{\operatorname{RSSI}}$ and $\sigma^{2}$ is the variance and defined by the formula (7):

$$
\sigma^{2}=\frac{1}{m-1} \sum_{\mathrm{i}=1}^{\mathrm{m}}\left(\mathrm{RSSI}_{\mathrm{i}}-\mu\right)^{2}
$$

The idea of Gaussian filter is to limit the RSSI within the range of one standard deviation $\sigma$ from the mean. This is explained in (8):

$$
\begin{aligned}
\mathrm{P}(\mu-\sigma & <\mathrm{RSSI}<\mu+\sigma) \\
& =\int_{\mu-\sigma}^{\mu+\sigma} \mathrm{f}(\mathrm{RSSI}) \mathrm{dRSSI} \approx 0.682
\end{aligned}
$$

Other RSSI values that outside the effective range will be removed.

\section{3) Kalman filter}

The Kalman filter [10] is proposed to cope with noise obeying the normal distribution. The basic principle of this filter is implementing a predictor and a corrector to minimize the error covariance. It will revise the past, present and future state which include noise to correct/predict the RSSI measurement. As stated, there are two main stages in Kalman filtering: prediction and updating and they are represented in equations (9-13):

\section{Prediction phase:}

State Model: $\quad \widehat{\mathrm{x}_{\mathrm{k}}}=\mathrm{Fx}_{\mathrm{k}-1}+\mathrm{B}_{\mathrm{k}-1} \mathrm{u}_{\mathrm{k}}$

Error covariance: $\widehat{\mathrm{p}_{\mathrm{k}}}=\mathrm{Fp}_{\mathrm{k}-1} \mathrm{~F}+\mathrm{q}_{\mathrm{k}}$

\section{Correction phase:}

Kalman Gain: $\mathrm{K}_{\mathrm{k}}=\widehat{\mathrm{p}_{\mathrm{k}}} \mathrm{H}\left(\mathrm{H} \widehat{\mathrm{p}_{\mathrm{k}}} \mathrm{H}^{\mathrm{T}}+\mathrm{r}_{\mathrm{k}}\right)^{-1}$

Updated covariance: $\mathrm{p}_{\mathrm{k}}=\left(\mathrm{I}-\mathrm{K}_{\mathrm{k}} \mathrm{H}\right) \widehat{\mathrm{p}_{\mathrm{k}}}$

Updated state: $\mathrm{x}_{\mathrm{k}}=\widehat{\mathrm{x}_{\mathrm{k}}}+\mathrm{K}_{\mathrm{k}}\left(\mathrm{z}_{\mathrm{k}}-\mathrm{H} \widehat{\mathrm{x}_{\mathrm{k}}}\right)$

where $\mathrm{F}$ is the state transition matrix; $\mathrm{Bu}$ is the control input if applied; $q$ is the system noise covariance; $I$ is the identity matrix; $r$ is the measurement noise covariance and $\mathrm{z}$ is the measurements or true observation.

\section{Distance estimation}

The distance between beacons and a device can be obtained using the log-normal shadowing and calibrated factor. From (2), the conversion between RSSI and distance can be expressed by the formula (14) bellow:

$$
\mathrm{d}=10^{\frac{\text { RSSI-A }}{10 \eta}}
$$

As highlighted above, all beacons and devices are considered static. Hence it is assumed that after the factor calibration and RSSI smoothing, there is no noticeable noise in the distance calculation.

\section{Positioning techniques}

\section{1) Trilateration - Centroid}

Trilateration will estimate the device's position from at least three surrounding beacons. Three anchors could be beacons or access points each at a specific distance $d$, which is represented as a circle with radius $d$. The actual position will be where the three circles intersect, which can be calculated by solving the three circle equations (15):

$$
\left\{\begin{array}{l}
d_{1}{ }^{2}=\left(x-x_{1}\right)^{2}+\left(y-y_{1}\right)^{2} \\
d_{2}{ }^{2}=\left(x-x_{2}\right)^{2}+\left(y-y_{2}\right)^{2} \\
d_{3}{ }^{2}=\left(x-x_{3}\right)^{2}+\left(y-y_{3}\right)^{2}
\end{array}\right.
$$

where $d_{1}, d_{2}, d_{3}$ are the distances from anchors to the user's device, $(\mathrm{x}, \mathrm{y})$ is the coordination of user's location $\mathrm{D}$ and $\left(\mathrm{x}_{1}, \mathrm{y}_{1}\right),\left(\mathrm{x}_{2}, \mathrm{y}_{2}\right),\left(\mathrm{x}_{3}, \mathrm{y}_{3}\right)$ are coordinate of three anchors A, B and C respectively. Fig. 4(a) describes the trilateration scheme.

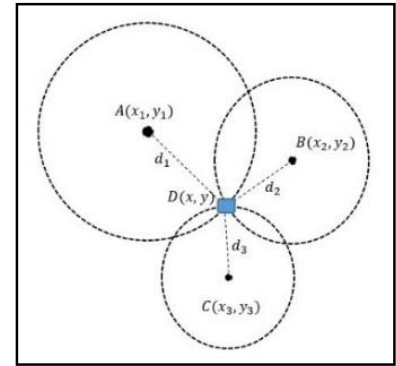

(a) Trilateration calculation

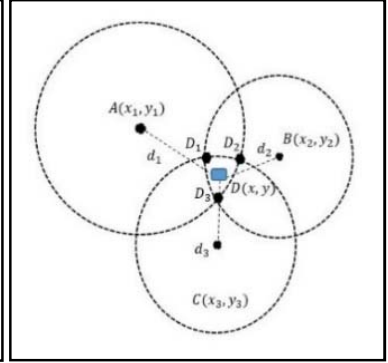

(b) Centroid calculation
Fig. 4. Trilateration technique

In practice, the RSSI smoothing process and distance calculation will produce some error. The worst case of scenario for trilateration will be that the three circles might not intersect at a single point. To address this issue, a simple method is used to estimate the position by finding the centroid of the intersected area. Fig. 4(b) shows this situation. The estimated coordination is calculated by finding the centroid of the polygon as given in (16):

$$
(x, y)=\left(\frac{x_{D_{1}}+x_{D_{2}}+x_{D_{3}}}{3}, \frac{y_{D_{1}}+y_{D_{2}}+y_{D_{3}}}{3}\right)
$$

where $(\mathrm{x}, \mathrm{y})$ is the coordination of device position $\mathrm{D}$, $\left(\mathrm{x}_{\mathrm{D}_{\mathrm{i}}}, \mathrm{y}_{\mathrm{D}_{\mathrm{i}}}\right)$ are the coordination of intersect points between two anchors' working range: $D_{1}$ is the intersection point between beacon $\mathrm{A}$ and $\mathrm{B}, \mathrm{D}_{2}$ is the intersection point between beacon $\mathrm{B}$ and $\mathrm{C}$ and $\mathrm{D}_{3}$ is the intersection point between beacon $\mathrm{C}$ and $\mathrm{A}$.

\section{2) Weighted Centroid}

In practical calculation of (15), a scenario may arise where the distance between two beacons is larger than the sum of distances from these beacons to device. For example, $L_{A B}>d_{1}+d_{2}$, i.e. the Euclidean distance between beacons $A$ and $B$ is larger than the sum of distances from $\mathrm{A}$ and $\mathrm{B}$ to the device. This leads to values in the imaginary quadrant, which in turn will require more complex processing, when finding the intersection. Furthermore, in the centroid calculation, all the beacons and devices are treated equally regardless of their distance from the device. Hence, it may lead to error in position calculation. The RSSI or the estimated distance can be used to overcome this issue of the Trilateration-Centroid approach without increasing the complexity.

The RSSI from each beacon to device shows the contribution of each beacon to the positioning. 
Furthermore, RSSI represents the relationship in terms of signal strength and distance between beacons and devices. Hence, the weighted factor $\mathrm{w}$ can be expressed with distance to represent the contribution of each beacon as formula (17):

$$
\mathrm{w}_{\mathrm{i}}=\frac{1}{\mathrm{~d}_{\mathrm{i}}{ }^{\omega}}
$$

where $\omega$ is the degree representing the contribution of each beacon. In our test bed, as the distance under $10 \mathrm{~m}$ so the $\omega$ is set to be 1 [14]. The weighted factor for $D_{1}$, $\mathrm{D}_{2}$ and $\mathrm{D}_{3}$ can be expressed as follows:

$$
\widetilde{\mathrm{w}_{1}}=\frac{1}{\mathrm{~d}_{1}{ }^{\omega}+\mathrm{d}_{2}{ }^{\omega}} ; \widetilde{\mathrm{w}_{2}}=\frac{1}{\mathrm{~d}_{2}{ }^{\omega}+\mathrm{d}_{3}{ }^{\omega}} ; \widetilde{\mathrm{W}}_{3}=\frac{1}{\mathrm{~d}_{3}{ }^{\omega}+\mathrm{d}_{1}{ }^{\omega}}
$$

However, in these weighted distances, if one distance is much larger than another, the beacon with the smaller distance to the device might be considered as unimportant. Each beacon should play equal role in defining device position. Hence, resolving (16) and (18), the weighted centroid algorithm is modified in (19) as agreed with [13]:

$$
\mathrm{w}_{1}=\frac{1}{\mathrm{~d}_{1}{ }^{\omega}}+\frac{1}{\mathrm{~d}_{2}{ }^{\omega}} ; \mathrm{w}_{2}=\frac{1}{\mathrm{~d}_{2}{ }^{\omega}}+\frac{1}{\mathrm{~d}_{3}{ }^{\omega}} ; \mathrm{w}_{3}=\frac{1}{\mathrm{~d}_{3}{ }^{\omega}}+\frac{1}{\mathrm{~d}_{1}{ }^{\omega}}
$$

And the final position results in the following formula (20):

$$
\mathrm{x}=\frac{\sum_{\mathrm{i}=1}^{\mathrm{h}} \mathrm{x}_{\mathrm{D}_{i}} \mathrm{w}_{\mathrm{i}}}{\sum_{\mathrm{i}=1}^{\mathrm{h}} \mathrm{w}_{\mathrm{i}}} ; \mathrm{y}=\frac{\sum_{\mathrm{i}=1}^{\mathrm{h}} \mathrm{y}_{\mathrm{D}_{\mathrm{i}}} \mathrm{w}_{\mathrm{i}}}{\sum_{\mathrm{i}=1}^{\mathrm{h}} \mathrm{w}_{\mathrm{i}}}
$$

where $h$ is the number of intersection points.

\section{3) Least Square Estimation}

Least Square Estimation (LSE) is a well-known method to solve the lack of GPS for positioning objects [11]. It is based on the following equation (21):

$$
\mathrm{y}=\mathrm{Mx}+\epsilon
$$

The approach is to choose an appropriate value for estimated $\hat{x}$ so that the norm value $\|\phi \hat{x}-y\|$ is minimized. Then the general linearized solution is:

$$
M \hat{x} \approx \beta
$$

Applying into the classic positioning problem, the matrices can be represented as in equations (23-25):

$$
\begin{gathered}
M=2\left[\begin{array}{ll}
\mathrm{x}_{1}-\mathrm{x}_{2} & \mathrm{y}_{1}-\mathrm{y}_{2} \\
\mathrm{x}_{1}-\mathrm{x}_{3} & \mathrm{y}_{1}-\mathrm{y}_{3} \\
\mathrm{x}_{1}-\mathrm{x}_{4} & \mathrm{y}_{1}-\mathrm{y}_{4}
\end{array}\right] \\
\beta=\left[\begin{array}{l}
\mathrm{d}_{2}^{2}-\mathrm{d}_{1}^{2}-\left(\mathrm{x}_{2}^{2}+\mathrm{y}_{2}^{2}\right)+\left(\mathrm{x}_{1}^{2}+\mathrm{y}_{1}^{2}\right) \\
\mathrm{d}_{3}^{2}-\mathrm{d}_{1}^{2}-\left(\mathrm{x}_{3}^{2}+\mathrm{y}_{3}^{2}\right)+\left(\mathrm{x}_{1}^{2}+\mathrm{y}_{1}^{2}\right) \\
\mathrm{d}_{3}^{2}-\mathrm{d}_{1}^{2}-\left(\mathrm{x}_{3}^{2}+\mathrm{y}_{3}^{2}\right)+\left(\mathrm{x}_{1}^{2}+\mathrm{y}_{1}^{2}\right)
\end{array}\right] \\
\hat{\mathrm{x}}=\left[\begin{array}{l}
\mathrm{x} \\
\mathrm{y}
\end{array}\right]
\end{gathered}
$$

The solution is given by:

$$
\hat{\mathrm{x}}=\left(\mathrm{M}^{\mathrm{T}} M\right)^{-1} \mathrm{M} \beta
$$

\section{4) Improved Least Square Estimation}

In the classic LSE, it uses the position $(0,0)$ as the initialization. The main idea of this improvement is cooperating with the priori positioning result from trilateration - weighted centroid algorithm and finding the value of change in distance between two immediate states. Resolving equation (15), we find the Euclidean distance between the beacon and device, which is represented as a function of $\mathrm{x}$ and $\mathrm{y}$, shown below:

$$
d_{i}^{k}=f\left(x^{k}, y^{k}\right)=\sqrt{\left(x^{k}-x_{i}\right)^{2}+\left(y^{k}-y_{i}\right)^{2}}
$$

where $d_{i}^{k}$ is the distance from beacon $i$ to device at the time state $\mathrm{k}\left(\mathrm{x}^{\mathrm{k}}, \mathrm{y}^{\mathrm{k}}\right)$ is the estimate position and $\left(\mathrm{x}_{\mathrm{i}}, \mathrm{y}_{\mathrm{i}}\right)$ is the beacon i position.

Using Taylor expansion to find the distance in the next time state $\mathrm{k}+1$ :

$$
\begin{aligned}
& \mathrm{f}\left(\mathrm{x}^{\mathrm{k}+1}, \mathrm{y}^{\mathrm{k}+1}\right)=\mathrm{f}\left(\mathrm{x}^{\mathrm{k}}, \mathrm{y}^{\mathrm{k}}\right) \\
& +\frac{\partial \mathrm{f}\left(\mathrm{x}^{\mathrm{k}}, \mathrm{y}^{\mathrm{k}}\right)}{\partial \mathrm{x}^{\mathrm{k}}} \Delta \mathrm{x}^{\mathrm{k}}+\frac{\partial \mathrm{f}\left(\mathrm{x}^{\mathrm{k}}, \mathrm{y}^{\mathrm{k}}\right)}{\partial \mathrm{y}^{\mathrm{k}}} \Delta \mathrm{y}^{\mathrm{k}}
\end{aligned}
$$

As $f\left(x^{k}, y^{k}\right)$ is the first order function, the Taylor expansion results in (29):

$$
\begin{aligned}
d_{i}^{k+1}= & f\left(x^{k+1}, y^{k+1}\right) \\
& =d_{i}^{k}-\frac{x_{i}-x^{k}}{d_{i}^{k}} \Delta x^{k}-\frac{y_{i}-y^{k}}{d_{i}^{k}} \Delta y^{k}
\end{aligned}
$$

In this equation, $\Delta \mathrm{x}^{\mathrm{k}}=\mathrm{x}^{\mathrm{k}+1}-\mathrm{x}^{\mathrm{k}}$ and $\Delta \mathrm{y}^{\mathrm{k}}=\mathrm{y}^{\mathrm{k}+1}-$ $\mathrm{y}^{\mathrm{k}}$ are the change in distance between time $\mathrm{k}$ and $\mathrm{k}+1$. If either of these values is 0 , it can be considered as the classic Least Square problem.

For $\mathrm{n}$ beacons in 2D dimension and static devices, we can express (29) as a series of matrices representing the coordinates over the Euclidean distance, change in Euclidean distance and change in time states of coordinates (30-32).

$$
\begin{gathered}
M=\left[\begin{array}{cc}
\frac{x_{1}-x^{k}}{d_{1}^{k}} & \frac{y_{1}-y^{k}}{d_{1}^{k}} \\
\vdots & \vdots \\
\frac{x_{n}-x^{k}}{d_{n}^{k}} & \frac{y_{n}-y^{k}}{d_{n}^{k}}
\end{array}\right] \\
\beta=\left[\begin{array}{c}
d_{1}^{k+1}-d_{1}^{k} \\
\vdots \\
d_{n}^{k+1}-d_{n}^{k}
\end{array}\right] \\
\hat{x}=\left[\begin{array}{c}
\Delta x^{k} \\
\Delta y^{k}
\end{array}\right]
\end{gathered}
$$

This becomes a Least Square problem with solution of finding: $M \hat{x} \approx \beta$. Solving this problem gives us the change in distance between two immediate states.

\section{EXPERIMENTAL RESULTS}

\section{A. Test bed setup}

Fig. 5 shows the test bed environment for our experimentation. Four iBeacons are placed in a grid area of $5 \times 5$ meters. They are placed at fixed positions as followed: iBeacon_1 (coordination 0,0$)$, iBeacon_2 (0, $5)$, iBeacon_3 $(5,5)$ and iBeacon_4 $(5,0)$. There are five different positions of devices Position A $(0,3)$, Position B $(2,4)$, Position C $(1,1)$, Position D $(3,2)$, Position E $(5,5)$. There are ten tables randomly placed around the testbed but all devices can see each other directly. Table I lists devices used in our experiment for measurements.

There are several assumptions made for this experimentation listed as below:

- All the devices are static.

- All the antennas are omnidirectional. 
- All the devices are at the same height of $1.2 \mathrm{~m}$.

- Radio frequency interferences are negligible.

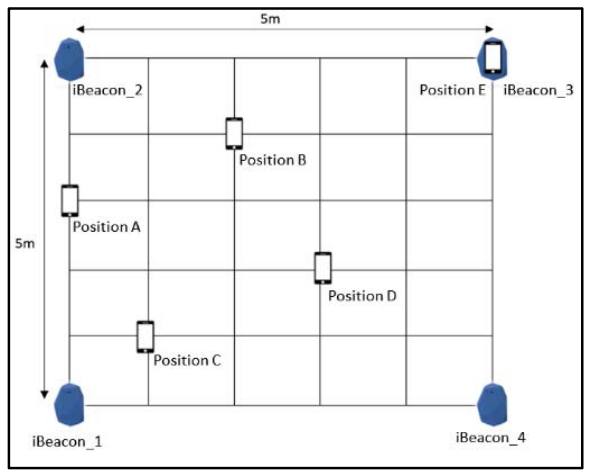

Fig. 5. Testbed setup

TABLE I. DEVICES

\begin{tabular}{|l|l|c|}
\hline \multirow{2}{*}{ Items } & \multicolumn{2}{|c|}{ Details } \\
\cline { 2 - 3 } & Manufacturer & Settings \\
\hline iBeacon & Estimote & $\begin{array}{c}\text { Transmit power: 4 dBm } \\
\text { Advertising interval: } 400 \mathrm{~ms}\end{array}$ \\
\hline iPad 2 & Apple & $\begin{array}{c}\text { iOS } 10.2 .1 \\
\text { Wi-Fi: On - Bluetooth: On }\end{array}$ \\
\hline
\end{tabular}

\section{A. Results and discussion}

\section{1) Calibration}

The RSSI measurements were collected 100 times using Estimote application at predefined locations and fixed distances varying from 0 to $11 \mathrm{~m}$ on the $\mathrm{iPad}$ device. Fig. 6 shows the calibration results.

The calibrated result gives the environment factor for our testbed as $\eta=2.6472$. The received signal strength at $1 \mathrm{~m}$ is $\mathrm{A}=-54.6476 \mathrm{dBm}$ comparing with the Estimote documentation [12], with transmission power of $4 \mathrm{dBm}$, in theory, A will be approximately $-60 \mathrm{dBm}$.

\section{2) RSSI smoothing}

At each position, the RSSI from each beacon were observed 100 times. The measurement noise and the system noise are $r=0.1$ and $q=0.1$ respectively. Fig. 7 shows the measurements for raw RSSI data and RSSI data after applying feedback filter, Gaussian filter and Kalman filter at 1 meter and 5 meters.

The results show that in general all filters help smoothing the RSSI measurement. At 1m, Gaussian filter gives the variance of 1.97 whereas Feedback filter gives the variance of 0.62 and Kalman filter gives the least variance at 0.11 . At $5 \mathrm{~m}$, Gaussian filter has 2.73 variance whereas Feedback filter and Kalman achieves 1.62 and 1.13 variance respectively. It can be seen that Kalman filter has the best performance out of the three filters. It smooths the RSSI very close to the mean value. This is because of the minimal noise in the measurement from the system due to the test bed conditions being close to free space. However, for real-life scenario where additional noises due to user's movement, interference, and environment conditions etc. will be present, the noise behavior needs be investigated further and re-modelled. This will be the next step in our research.

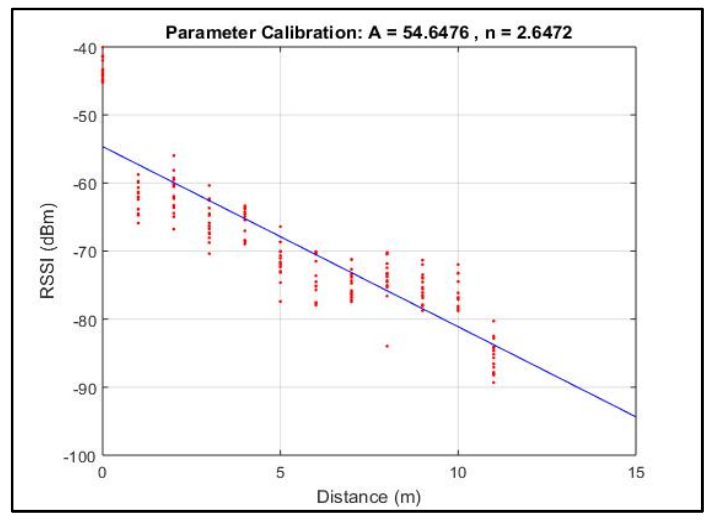

Fig. 6. Factor calibration

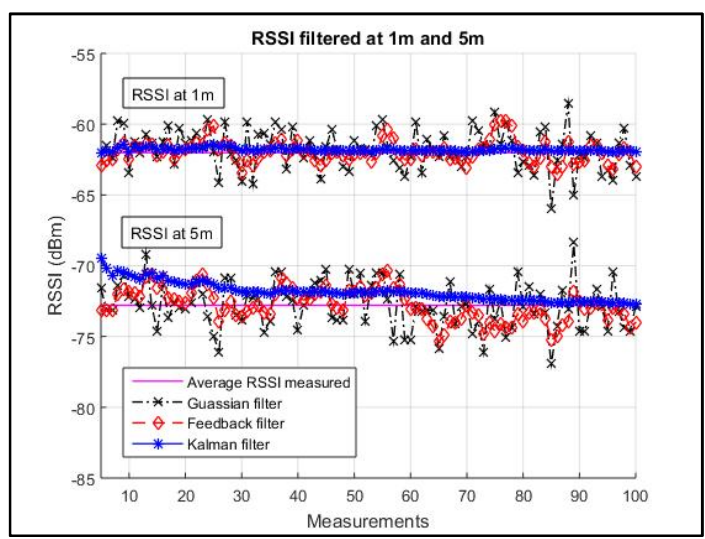

Fig. 7. RSSI filtered

\section{3) Position Estimation results}

Fig. 8 shows the experimental results for the three positioning calculations when applying Kalman filter for RSSI measurements. Table II compares the accuracy among approaches.

As can be seen, our proposed approach has the best performance of all three approaches in terms of positioning for static device in our specific testbed. The mean error for Trilateration-weighted Centroid is $0.375 \mathrm{~m}$. The mean error for classic LSE is $0.333 \mathrm{~m}$. The improved LSE performs the best with a mean error of $0.192 \mathrm{~m}$. This is the result of correcting factor and smoothing the RSSI value. Especially when the device is in the edge of the test bed or in the side of the testbed, the improved LSE method outperforms its competitors. In position $\mathrm{A}(0,3)$, our method gives the positioning result of $(-0.115,0.233)$ whereas Trilateration-weighted Centroid results is $(-1.009,3.196)$ and classic LSE results is $(0.8917,3.078)$. In position $\mathrm{E}(5,5)$, the Trilateration-Centroid and classic LSE results are $(5.382,5.394)$ and $(4.525,4.537)$ respectively. The improved LSE estimates $(4.804,4.807)$ as the coordinate. This is because in this position in our testbed, distances from beacons to the device are the farthest, it leads to more fluctuation in RSSI even with 
the filtering. Furthermore, at farther distances, even small changes in RSSI will result in larger error distance estimations. However, using improved LSE and by incorporating the trilateration-weighted centroid approach results as the initial guess, this error is minimized.

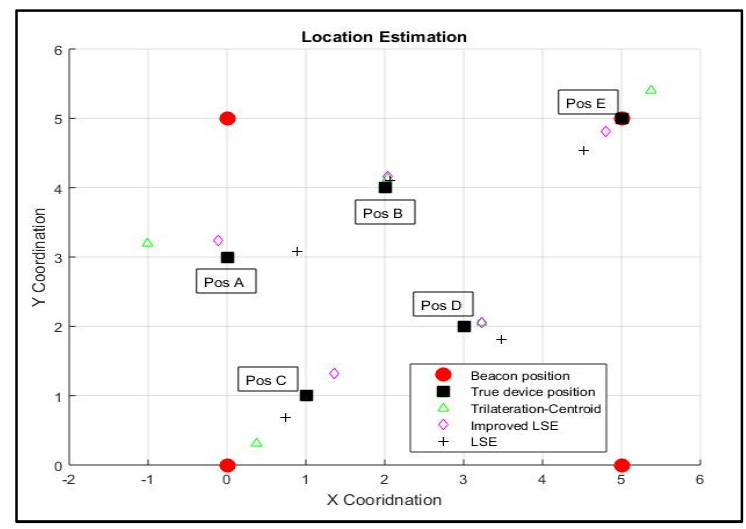

Fig. 8. Indoor positioning results

TABLE II. POSITIONING ACCURACY

\begin{tabular}{|l|l|l|}
\hline \multicolumn{1}{|c|}{ Method } & $\begin{array}{c}\text { Average } \\
\text { error }\end{array}$ & $\begin{array}{c}\text { Maximum } \\
\text { error }\end{array}$ \\
\hline Trilateration-weighted Centroid & $0.375 \mathrm{~m}$ & $1.009 \mathrm{~m}$ \\
\hline LSE & $0.333 \mathrm{~m}$ & $0.89 \mathrm{~m}$ \\
\hline Improved LSE & $0.192 \mathrm{~m}$ & $0.354 \mathrm{~m}$ \\
\hline
\end{tabular}

\section{FUTURE WORK AND CONCLUSION}

In this paper, an optimized indoor localization for static device was proposed using RSSI-based BLE technology. The factor calibration process was explained and several different RSSI filters were tested. Three different approaches namely, Trilaterationweighted Centroid, classic LSE and improved LSE for position estimation were discussed. We have shown that our positioning approach can achieve very high accuracy in terms of positioning for static device and its output is very promising. Experiments results also shows that the improved LSE method outperforms the other techniques with a high accuracy of about 0.2 $0.35 \mathrm{~m}$. As a next step in our research, dynamic devices and tracking application using our approach will be investigated. Also, there will be a remodeling in factor calibration for a more complicated indoor environment.

\section{REFERENCES}

[1] G. Dedes and A. Dempster, "Indoor GPS positioning challenges and opportunities", VTC-2005-Fall. 2005 IEEE 62nd Vehicular Technology Conference, 2005.
[2] K. Al Nuaimi and H. Kamel, "A survey of indoor positioning systems and algorithms", 2011 International Conference on Innovations in Information Technology, 2011.

[3] K. Townsend, Getting started with Bluetooth low energy. Beijing: O'Reilly, 2015.

[4] "iBeacon - Apple Developer", Developer.apple.com, $2017 . \quad$ [Online]. Available: https://developer.apple.com/ibeacon/.

[5] W. Wang, H. Ma, Y. Wang and M. Fu, "Localization of static target in WSNs with least-squares and extended Kalman filter", 2012 12th International Conference on Control Automation Robotics \& Vision (ICARCV), 2012.

[6] S. Gezici, I. Guvenc and Z. Sahinoglu, "On the Performance of Linear Least-Squares Estimation in Wireless Positioning Systems", 2008 IEEE International Conference on Communications, 2008.

[7] M. Rusli, M. Ali, N. Jamil and M. Din, "An Improved Indoor Positioning Algorithm Based on RSSITrilateration Technique for Internet of Things (IOT)", 2016 International Conference on Computer and Communication Engineering (ICCCE), 2016.

[8] J. Anuradha, S. Lokuliyana, D. Chathurangi and D. Vithana, "Indoor Positioning: Novel Approach for Bluetooth Networks using RSSI Smoothing", International Journal of Computer Applications, vol. 137, no. 13, pp. 26-32, 2016.

[9] S. Halder, P. Giri and W. Kim, "Advanced Smoothing Approach of RSSI and LQI for Indoor Localization System", International Journal of Distributed Sensor Networks, vol. 11, no. 5, p. 195297, 2015.

[10] S. Lee, I. Lim and J. Lee, "Method for Improving Indoor Positioning Accuracy Using Extended Kalman Filter", Mobile Information Systems, vol. 2016, pp. 1-15, 2016.

[11] I. Sharp and K. Yu, "Enhanced Least-Squares Positioning Algorithm for Indoor Positioning", IEEE Transactions on Mobile Computing, vol. 12, no. 8, pp. 1640-1650, 2013.

[12] Developer.estimote.com, 2017. [Online]. Available: http://developer.estimote.com/

[13] H. Shi, "A new weighted centroid localization algorithm based on RSSI," 2012 IEEE International Conference on Information and Automation, Shenyang, 2012, pp. 137 14

[14] J. Blumenthal, R. Grossmann, F. Golatowski and D. Timmermann, "Weighted Centroid Localization in Zigbee-based Sensor Networks," 2007 IEEE International Symposium on Intelligent Signal Processing, Alcala de Henares, 2007, pp. 1-6. 